\title{
Challenges for First Time GIS User among Student Teachers
}

\author{
Mengieng Ung, \\ ${ }^{a}$ Humanities and Social Studies Education, National Institute of Education/Nanyang Technological University, Singapore \\ mengieng.ung@nie.edu.sg
}
Keywords: Geography Education, Pre-service and In-service Student Teachers, and National Institute of Education, Singapore

Abstract:

National Institute of Education (NIE), Singapore is a national teacher education institute with a mission to excel in teacher education and educational research. It is where student teachers in Singapore are trained. Its program consists of undergraduate, higher degrees and professional learning. Geography as a discipline is offered under the Humanities and Social Studies Education (HSSE) an academic group at NIE.

An Introduction to GIS course has been offered to second-year pre-service undergraduate and the higher degree level in-service Geography student teachers (STs) at HSSE/NIE. There are a total of 14 STs (eight pre-service and six inservice) aged between 19 and 32 years old. Pre-service STs will become a primary school, secondary school or junior college teachers once they graduate from the undergraduate program from NIE. In-service STs are currently teachers at the above-mentioned education institutions. They are returning to NIE for the higher degree program. They all had no prior experience with GIS. Key GIS theories and techniques including data models, map projection and GPS, spatial join, geo-processing, geo-referencing and digitizing were introduced to them throughout the course.

Assignments were given to students to work individually as part of the assessment components. Assignments asked them to create thematic maps showing spatial and temporal distribution of the world's total fertility rates (TFR), issues in Economic Geography and Education related issues by applying GIS theories and techniques learned during the lectures. Upon submitting each assignment, STs were asked to indicate the challenges encountered while completing the assignments and suggestions to solve their problems.

The major set challenge for STs was downloading, cleaning, joining table and understanding the attribute tables. STs, especially those who had limited prior experience working with the dataset, found the above-mentioned tasks to be problematic and confusing all together. Those STs had a hard time applying those steps when they need to do the assignment, which required starting the process from scratch.

In order to avoid under or over-representation, almost all STs expressed major challenges when it comes to choosing color and number of classes for choropleth maps. STs further emphasized that it required them to know beyond GIS skills in order to make thematic maps meaningfully. For instance, in order to showcase TFR, one needs to understand that TFR of 2.1 is the replacement rate, TFR of 1.5 or below is considered low or in danger in terms of population growth. Therefore, one needs to take those factors into consideration when choosing the cutoff points and the total number of classes.

To overcome those challenges, a series of suggestions were provided by STs. For instance, more practices and more exercises of the same nature, pair or group work instead of individual work, allocate more time for each assignment, both instruction manual and video tutorials are needed.

This exercise pedagogically provides both STs and me, the instructor, a fresh perspective when it comes to teaching GIS to students. As an instructor, I need to strike the balance between concepts and practices. Furthermore, I need to take student's profiles and prior knowledge into account when planning my lectures in order to leave no one behind. Understanding both concepts and practices of GIS in crucial for STs because they will be teaching GIS upon completing the course. Integration of GIS into primary school, secondary school and junior college will be part of Singapore's smart nation initiative. 